\title{
Object Tracking Using Computer Vision Techniques
}

\author{
N. Sushmitha ${ }^{1}$, B. Meena ${ }^{2}$, Mr.K.V Sriharsha ${ }^{3}$, Dr N.V Rao ${ }^{4}$ \\ ${ }^{1}$ CVR College of Engg /CSE Dept, Hyderabad, India \\ Email:sushuma.shiri@gmail.com \\ ${ }^{2}$ CVR College of Engg /CSE Dept, Hyderabad, India \\ Email:meenareddy38@gmail.com \\ ${ }^{3}$ CVR College of Engg /I.T Dept, Hyderabad, India \\ Email: sriharsha.phd@gmail.com \\ ${ }^{4}$ CVR College of Engg/CSE Dept, Hyderabad, India \\ Email:nvr@ieee.org
}

\begin{abstract}
Computer vision basically deals with different factors such as modeling of camera, lighting, color, texture, shape and motion that affect images and videos from visual inputs. Object detection and tracking are important components in many computer vision applications including activity recognition, traffic monitoring and automotive safety. This paper is about locating a moving object (or multiple objects) over a time using a stationary camera and associating the target object detections in consecutive video frames. In this perspective a video is captured by digital camera and is used for motion analysis. In the first stage of experiment frame differencing algorithm is chosen for object detection and its motion is estimated by associating the centroid of the moving object in each differenced frame. In the second stage of experiment same algorithm is chosen for object detection but motion of each, track is estimated by Kalman filter. However the best estimate is made by combining the knowledge of prediction and correction mechanisms that were incorporated as part of Kalman filter design. The tracking results obtained from two stages of experiment are presented for discussion.
\end{abstract}

Index Terms- computer vision, object tracking, centroid, image filtering, grey thresholding, morphological, dilation, erosion, trajectory, point tracking, Kalman filter, dynamic model, observation model

\section{INTRODUCTION}

Earlier computer vision systems [1] are only concern with scene analysis. The scene analysis is about recognizing the activities in a scene and to assimilate information from the sequence of images as a whole. There are two types of scene analysis one is static and the other is dynamic. Static scene analysis is about recognizing and understanding the activities of static objects from a stationary camera. There is a little scope of applying static scene analysis in the current day applications. Nowadays while observing a scene, the activities will never be static, they will be changing or moving rapidly with in a fraction of seconds. In such case there is a necessity to perform dynamic scene analysis. The changes in the scene may arise due to motion of camera or object. However system must be able to detect and track the changes that are going on rapidly over stationary or non stationary background. The dynamic scene analysis can be done in three cases stationary camera among moving objects (SCMO), Moving camera among stationary objects (MCSO), moving camera among moving objects (MCMO). As the researcher goal is to identify the objects of interest, track the motion, compute the characteristics of motion, SCMO gained much attention in this regard. In our paper the discussion is confined to stationary camera among moving objects. The analysis is about interpreting the activity of the scene by indentifying the objects of interest, classifying them and estimate the trajectory of object in the image plane as it moves around the scene. For tracking an object over a scene it is essential to represent [2] an object either using a shape or appearance. There are different ways in which an object can be represented by shape; one way is representing an object by a point (centroid), or a set of points. The other representations are primitive geometric shapes such as rectangle, ellipse are used for tracking rigid and non rigid objects. There are also other categories of representations like boundary of an object (contour) and region inside the object (Silhouette) which are used for tracking complex non rigid shapes. As point representations are much more suitable for 
tracking objects that occupy small regions in a space, they are used for dynamic scene analysis in our experiment. Appearance based representation is also enormously used in many computer vision applications especially for tracking objects accurately in a scene. Template is one among which is formed using geometric shapes or silhouettes. The well and extensively used template matching for tracking objects in successive fames uses template representation. This approach works well for the cases where position and orientation simply called as pose; do not vary while tracking the objects in successive frames .The other is probability density based. These may be parametric or non parametric. Parametric take 1D Gaussian distribution, n-D Gaussian distribution or Gaussain Mixture Models (GMM). The objective parametric probabilities density based representation is to characterize an image region by its statistics. If the statistics differ from background, tracking should be enabled. Coming to Non parametric representations the most widely used for target representation is color histogram, which is mathematical equivalent to discrete probability density function of target region.

\section{OBJECT DETECTION}

Whenever objects appear in a video for tracking, they require object detection mechanism. It is the first step for dynamic scene analysis. It includes image segmentation process where foreground objects are separated from background objects by applying thresholding techniques. There is a possibility that foreground pixels may be affected with noise where image filtering is required for noise removal. The moment filtered foreground pixels are obtained; they are grouped into connected regions. The connected regions are further used to extract individual object features such as bounding box, area, perimeter etc.

\section{DIFFERENT TYPES OF OBJECT DETECTION MECHANISMS}

Object detection [3] can be achieved by using a representation of a scene called background model and the comparing the model with each incoming frame. Any significant change observed in the image from the background model is considered as moving object and is processed further for analysis. This method is known as background subtraction .It is a popular pre processing step used in computer vision applications as it allows detections of objects of interest in stationary and non stationary background even when the camera is fixed. However the subtraction results in large number of false detections with the changes in illumination and fine scale motion in the scene. There are also statistical based detection methods like adaptive background mixture modeled by mixture of Gaussians. In order to know whether a pixel belong to foreground or background, Gaussian distribution of mixture model for that pixel is evaluated. The most simple one is temporal differencing (or frame differencing) takes pixel wise difference between two or more consecutive difference. This method is unable to detect the foreground object when texture is non uniform and when its speed is changing. However in our experiment We have chosen this method in order to avoid false detections.

\section{FIRST STAGE OF EXPERIMENT}

\section{A. Algorithm for object detection and tracking}

In the first stage of experiment in order to identify the moving object in successive frames, frame differencing technique is applied. In this technique [4] a current frame is always subtracted from the previous frame by using image subtraction operator. The resulting differenced image is transformed to binary image as and when grey thresholding is applied. For removal of blur [5] (considered as Gaussian noise) in differenced image due to linear motion or unfocussed optics, filtering operation is applied. As it is known that Wiener filter is suitable for reconstruction of original from the noisy image and hence it is chosen for image filtering operation. Finally dilation and erosion operations are applied for removal of image imperfections. For each of the moving object that is identified in the preprocessed image, the centroid is computed. This centroid represents the moving object in each of the differenced image. Finally a trajectory is drawn by connecting the centroids of all differenced images [6]. The whole process is summarized in the following flowchart 


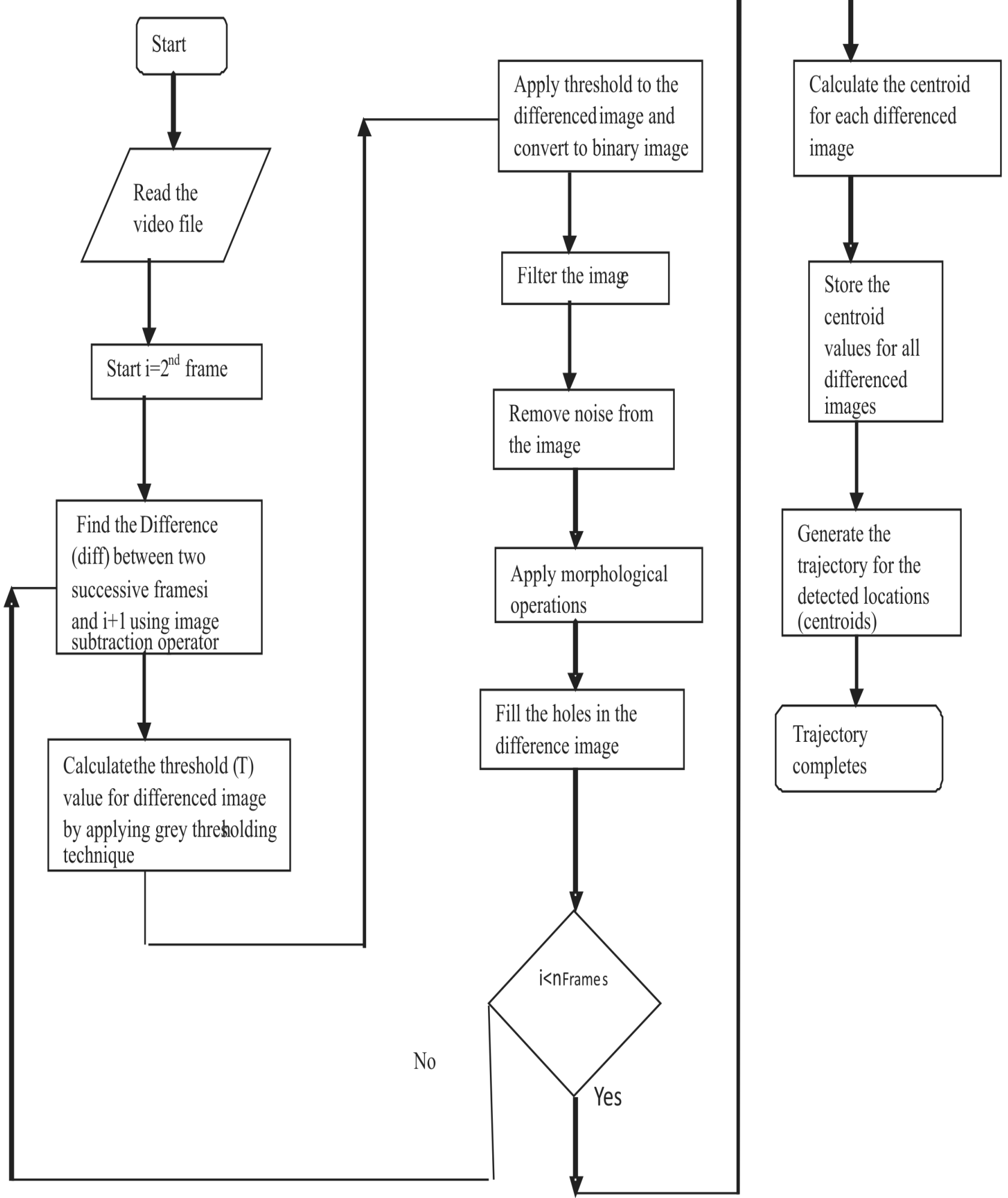




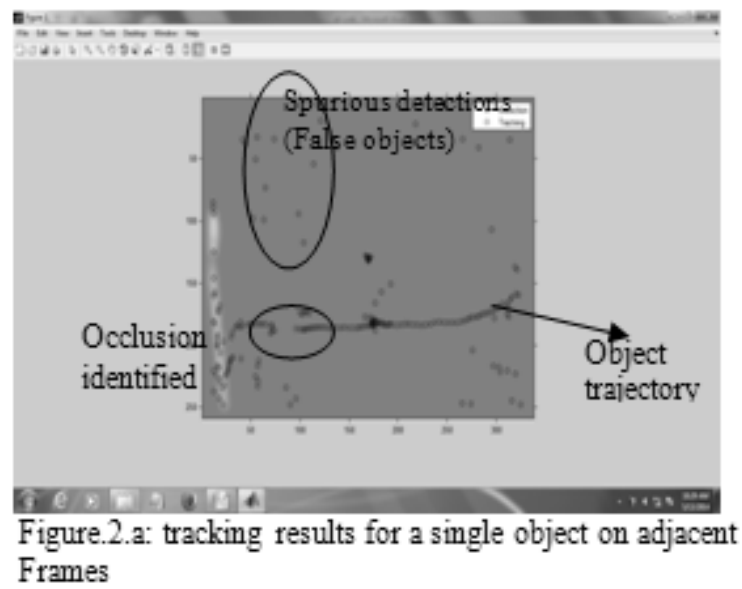

The results obtained from fig 2.a plotted on the graph With frame number on $\mathrm{X}$ axis and number of objects on Y axis

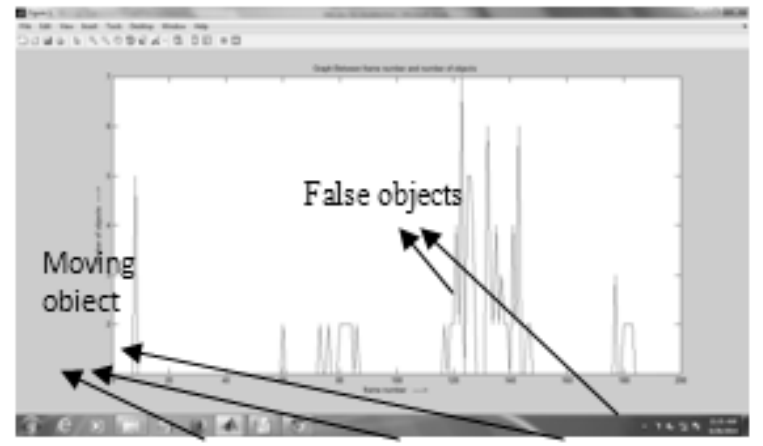

Figure2.b: graph showing moving object along with spurious detection

The algorithm is again applied on odd number of frames and results obtained as follows.

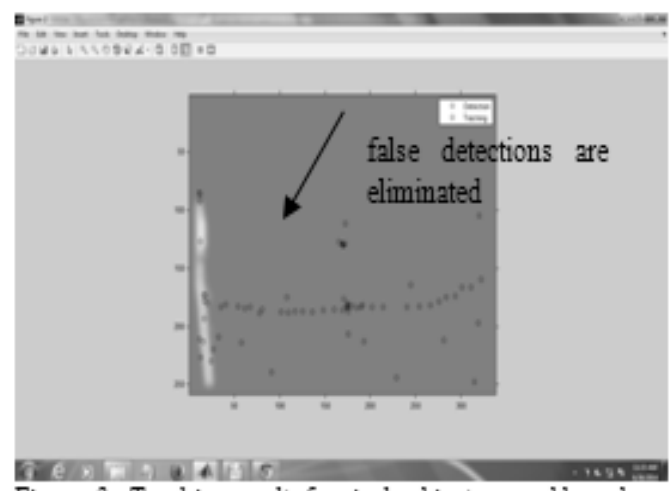

Figure. 3: Tracking results for single object over odd number of frame

\section{CLASSIFICATION OF OBJECT TRACKING}

Object tracking [7] can be categorized as point tracking, kernel based and silhouette based. Point tracking require object detection in every frame whereas kernel based and silhouette tracking only requires object detection when object first appear in the scene. In our paper the discussion is confined to point tracking .In this objects that are detected in successive or odd number of frames are represented by points. With an association of centroid of moving object over successive frames which includes object position and motion. Point tracking algorithms are either deterministic or statistical. Measurements obtained from video scene may be affected with noise and also object motion may undergo random perturbations. These are the challenges that are addressed in the deterministic methods. Statistical methods remove these uncertainties while estimating the state of an object in associated detections. Kalman filter falls under this category which can be applied to stationary and non stationary environments.

\section{SECOND STAGE OF EXPERIMENT}

\section{A. Kalman filter for object tracking}

Kalman filter [8] is s recursive predictive filter where each updated estimate of the state is computed from the previous estimate and the new input data. Hence only previous estimate requires storage. It eliminates the need for storing the entire past observed data. It is computationally more efficient than computing the estimate directly from the entire past observed data at each step of filtering process. In the previous case tracking results are not precise because of lack of prediction and correction mechanisms. Kalman filter make a good estimate by predicting the state of an object with dynamic model and correcting its state with observation model. Thus error covariance of the estimator is minimized by giving accurate tracking result. 


\section{B. Algorithm for frame differencing using Kalman filter}

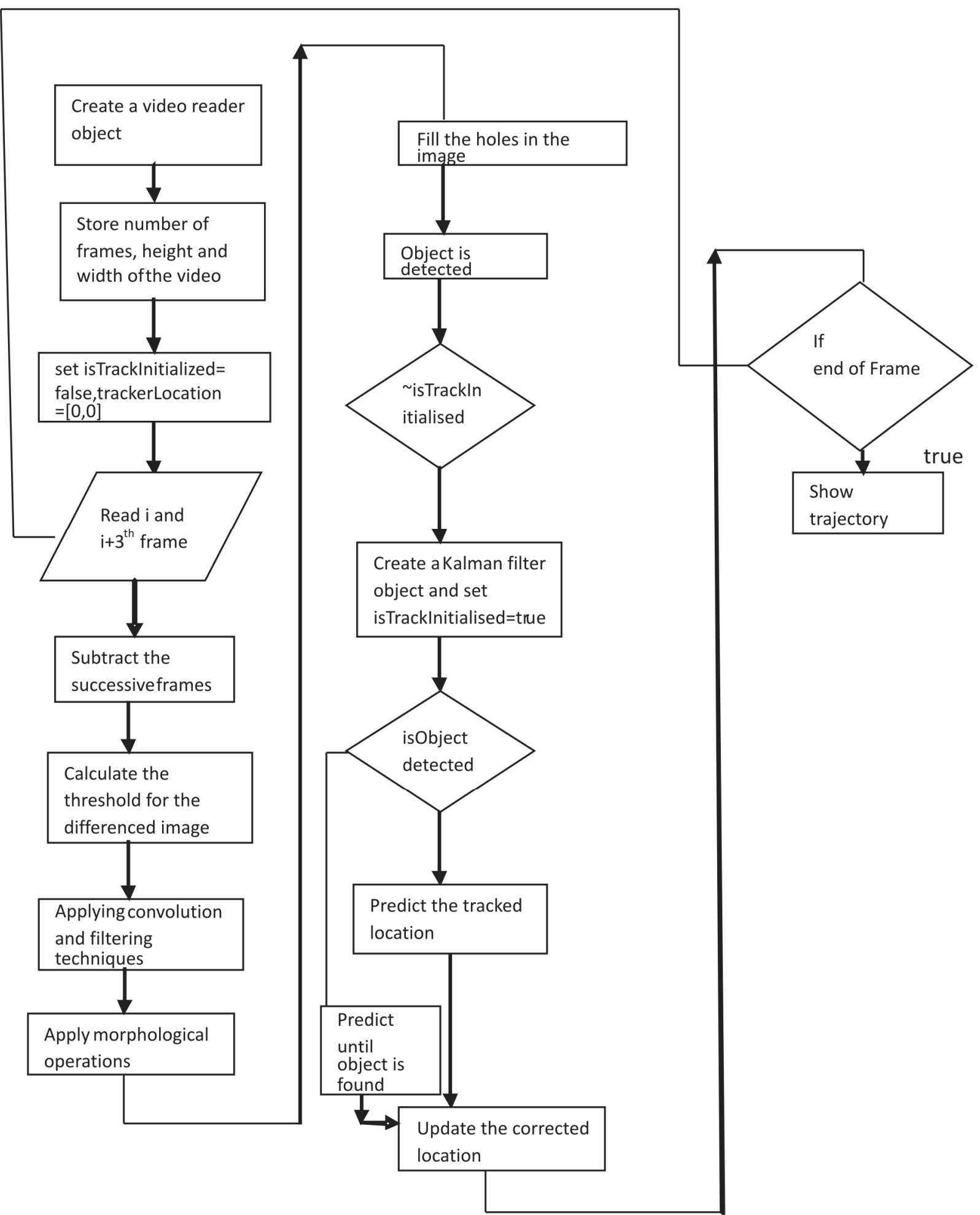

Figure.4: Object detection tracking using Kalman filter 


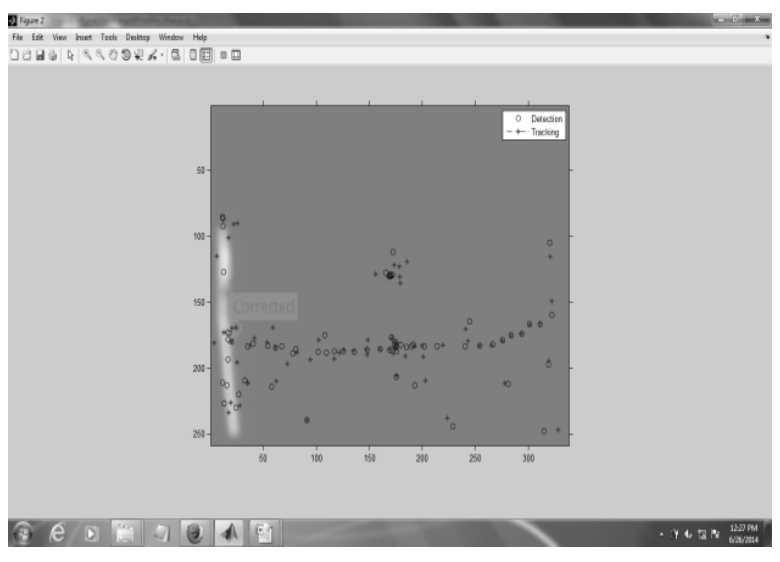

Figure.5: object detection and tracking using Kalman filter

From the fig. 5, it is observed that accurate tracking results were obtained with implementation of prediction and correction mechanism when compared to fig. $2 \& 3$.

\section{CONCLUSION}

In the first stage of experiment, moving object is detected and tracked successively by applying the algorithm on adjacent and odd number of frames. From the results obtained it is observed that comparatively less number of spurious detections were found by applying frame differencing algorithm on odd number of frames. As it was found that tracking results are not accurate in the first stage of experiment, Kalman filter is implemented and the path of the moving object is predicted and corrected.

\section{FUTURE WORK}

In this paper the discussion is confined to tracking single objects from a stationary camera, so the work is to be extended further on tracking multiple objects. The experiment is to be continued on moving object when camera is stationary and moving and at the same time it should possess the ability to cope up with complexities such as moving and changing objects, changing illumination and changing view points.

\section{REFERENCES}

[1] Ramesh Jain , Rangachar Kasturi, Brian G.Schunck , Machine Viraw-Hill , Inc., ISBN 0-07-032018-7,1995

[2] A.Yilmaz, O . Javed , and M.Shah ,"object tracking: A srvey", ACM Computing Surveys, Vol. 38,No-4, Article 13, December 2006.

[3] N Prabhakar , V . Vaithiyanathan, Akshaya Prakash Sharma , Anurag Singh and Pulkit Singhal ,'Object Tracking using Frame Differencing and Template matching", Research Journal of Applied Sciences , Engineeering and Technology 4(24):5497- 5501, Decemeber 15,2012.

[4] Macros martin-Fernandez, Emma Mnoz-Moreno, Carlos alberola-Lopez, "A Speckle Removal Filter Based on Anisotropic Wiener Filtering and the Rice Distribution", IEEE Ultrasonics Symposim,2006.

[5] Rasit Koker, Serap Cakar, Cemil Oz, "Moving Object detection and Target Prediction in Video Image Using Computer Vision “ ,proceedings of International Conference on Signal Processing, IJCI ,2003.

[6] Himani S.Parekh ,Darshak G.Thakore ,Udesang K. Jaliya ,"A survey on Object Detection and Tracking Methods", International Journal of Innovative research in Computer and Communication Engineering, Vol.2,Issue 2,Febrary 2014.

[7] Ramsey Faragher, "Understanding the Basis of the Kalman Filter via a Simple and Intuitive Derivation", IEEE Signal Processing Magazine, September 2012 